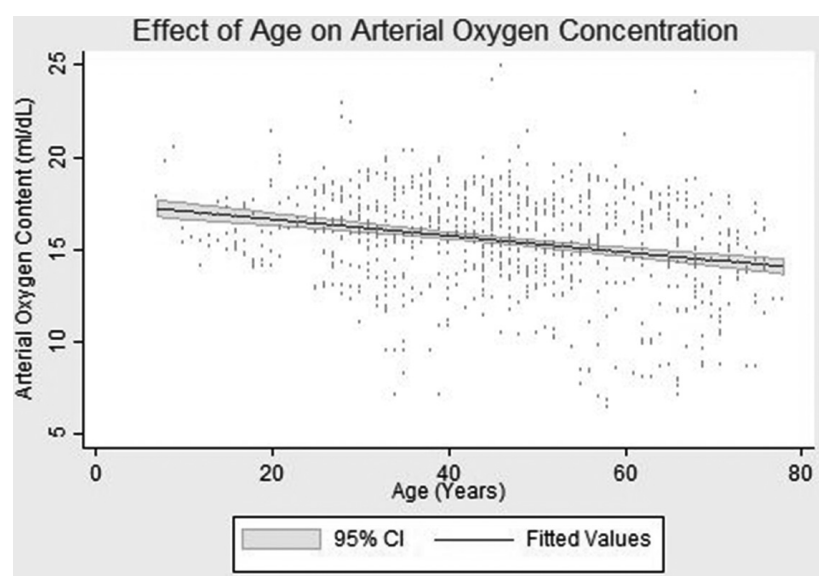

Abstract S52 Figure 1

Conclusions The body maintains arterial oxygen content within a normal range using well-known erythropoeitic mechanisms in response to hypoxaemia. Despite this feedback mechanism, in patients with pulmonary arteriovenous malformations, overall arterial oxygen content still decreases with age.

\section{Advances in cystic fibrosis}

\section{S53 OUTCOMES FOLLOWING BRONCHIAL ARTERY EMBOLISATION FOR HAEMOPTYSIS IN ADULTS WITH CYSTIC FIBROSIS}

${ }^{1}$ WG Flight, ${ }^{2} \mathrm{PJ}$ Barry, ${ }^{2} \mathrm{RJ}$ Bright-Thomas, ${ }^{2} \mathrm{~S}$ Butterfield, ${ }^{2} \mathrm{R}$ Ashleigh, ${ }^{2} \mathrm{AM}$ Jones. ${ }^{2}$ Oxford University Hospitals NHS Trust, Oxford, UK; ' ${ }^{2}$ University Hospital of South Manchester NHS Foundation Trust, Manchester, UK

\subsection{6/thoraxjnl-2015-207770.59}

Introduction Bronchial artery embolisation (BAE) is recommended as the therapy of choice for massive haemoptysis in cystic fibrosis (CF) but there are no randomised controlled trials of BAE in this setting. Outcomes from BAE are uncertain and the efficacy of BAE in sub-massive haemoptysis is unclear. We performed a single-centre observational study to investigate the role of BAE in CF-related haemoptysis.

Methods All patients with CF undergoing BAE from March 2011 to January 2015 were identified at the time of the procedure. Patient records were reviewed following hospital discharge or death. Severity of haemoptysis was classified as: massive ( $>240 \mathrm{ml} / 24 \mathrm{~h}$ or $>100 \mathrm{ml} /$ day for $\geq 2$ days), severe $(>20 \mathrm{ml} /$ $24 \mathrm{~h}$ ) or mild $(<20 \mathrm{ml} / 24 \mathrm{~h})$. Data were collected on adjuvant therapies, time to recurrence, complications and survival.

Results Twenty-seven patients underwent 49 BAE procedures. Median age was 30 years (range 18-72) and 16 (59\%) were male. Mean baseline FEV- ${ }_{1} \%$-predicted was $51.0 \%$ (SD 19.3). BAE was indicated for massive haemoptysis in 18 episodes (37\%), severe in $27(55 \%)$ and mild in 4 (8\%). Adjuvant therapies included tranexamic acid in $48(98 \%)$, intravenous antibiotics in 47 (96\%), intravenous vitamin $\mathrm{K}$ in 31 (63\%), ethamsylate in $8(16 \%)$, terlipressin in $7(14 \%)$ and propranolol in $5(10 \%)$ of episodes.

Twenty-nine (59\%) BAEs were complicated by adverse events including chest pain (29\%), headache (12\%), paraesthesia (10\%), groin pain $(6 \%)$, limb weakness $(4 \%)$ and limb ischaemia (2\%).
Eight patients (30\%) required $\geq 2$ BAEs during the study (range 2-7). Median time to first repeat BAE was 213 (range 18-682) days. Overall, haemoptysis recurred after 31/49 (63\%) procedures with no significant difference between massive and submassive haemoptysis $(61.1 \%$ vs $64.5 \%)$.

Five patients $(18.5 \%)$ died during the study and this group had a median $\mathrm{FEV}_{1} \%$-predicted of $32 \%$ (range 28-82\%). Mortality was $3.7 \%$ at 30 days following first BAE and $11.1 \%$ at six months. Four out of $8(50 \%)$ patients requiring repeat BAE died compared with $1 / 19(5 \%)$ who needed a single BAE only ( $\mathrm{p}=$ 0.006).

Conclusion BAE may be life-saving but is associated with considerable morbidity in CF. Need for repeat BAE is associated with increased mortality.

\section{S54 COGNITIVE FUNCTION IN ADULTS WITH AND WITHOUT CYSTIC FIBROSIS RELATED DIABETES (CFRD) ATTENDING A LARGE UK CYSTIC FIBROSIS UNIT}

${ }^{1}$ HK Chadwick, ${ }^{2} \mathrm{AM}$ Morton, ${ }^{2} \mathrm{~A}$ Driffill, ${ }^{2} \mathrm{~A}$ Wood, ${ }^{2} \mathrm{~L}$ Gillgrass, ${ }^{1} \mathrm{~L}$ Dye, ${ }^{1} \mathrm{CL}$ Lawton, ${ }^{2} \mathrm{MW}$ Mansfield, ${ }^{2} \mathrm{DG}$ Peckham. 'School of Psychology, University of Leeds, Leeds, UK; ${ }^{2}$ Regional Adult Cystic Fibrosis Unit, St James' Hospital, Leeds, UK

10.1136/thoraxjnl-2015-207770.60

Introduction and objectives On reaching adulthood many cystic fibrosis (CF) sufferers develop cystic fibrosis related diabetes (CFRD). CFRD shares clinical characteristics with type 1 (T1DM) and type 2 diabetes mellitus (T2DM). Impaired glucose tolerance (IGT), T1DM and T2DM have deleterious effects on cognitive performance. Hence, patients with CFRD are hypothesised to show similar impairment. This study aimed to elucidate the nature and severity of any cognitive impairment in patients with CFRD compared to non-diabetic patients with CF and healthy controls matched as closely as possible for age, gender and education level. Patients with CF were also matched as closely as possible on CFTR genotype.

Methods Adult (>16 years old), pancreatic insufficient patients registered to a large UK CF unit who had adequate verbal and written English were eligible. 49 patients with insulin-treated CFRD and 49 CF non-diabetics who had received a normal oral glucose tolerance test (OGTT) within the past 12 months were recruited. 46 healthy matched controls were recruited from relatives of patients and the general population. Cognitive performance was assessed using the Cambridge Neuropsychological Test Automated Battery (CANTAB). Subjective measures of sleep, stress, mood and cognitive functioning were also collected.

Results Matched controls performed better than both groups of patients with CF on tests of visual memory and learning, verbal memory, visual sustained attention, processing speed and executive function. Patients with CFRD performed significantly worse than controls on tests of mental flexibility and processing speed, which is consistent with the pattern of impairment shown in T1DM, and on verbal memory and learning, which is consistent with the pattern of impairment shown in T2DM. Compared to non-diabetic patients with CF, those with CFRD performed worse on tests of visual sustained attention, verbal memory, working memory, and processing speed.

Conclusion CFRD has a negative impact on cognitive performance akin to T1DM and T2DM. Non-diabetic patients with CF also show impaired cognition but to a lesser degree than CFRD. Even modest cognitive impairment in adults with CF may impact upon their self-management skills, health and quality of life. 